\title{
A UNIFIED BOUNDARY INTEGRAL EQUATION METHOD FOR A CLASS OF SECOND ORDER ELLIPTIC BOUNDARY VALUE PROBLEMS
}

\author{
M. REZAYAT, F. J. RIZZO and D. J. SHIPPY'
}

(Received 10 February 1983; revised 24 June 1983)

\begin{abstract}
A generalized integral equation formulation and a systematic numerical solution procedure are presented for a class of boundary value problems governed by a general second-order differential equation of elliptic type. Diverse numerical examples include problems of plane-wave scattering, three-dimensional fluid flow, and plane heat transfer for a body with a moving flame boundary. The last example employs certain representation functions useful to increase solution effectiveness in problems with an isolated integrable singularity.
\end{abstract}

\section{Introduction}

The partial differential equation

$$
A_{\alpha \sigma} \frac{\partial^{2} \phi}{\partial x_{\alpha} \partial x_{\sigma}}+B_{\alpha} \frac{\partial \phi}{\partial x_{\alpha}}+C \phi=0, \quad \alpha, \sigma=1,2, \ldots, n,
$$

in which $A_{\alpha \sigma}, B_{\alpha}$ and $C$ are constants satisfying the ellipticity requirement (cf. [21]), may be reduced to the canonical form

$$
\nabla^{2} \phi+\kappa \phi=0
$$

where $\kappa$ is a constant, $\phi=\phi\left(x_{1}, x_{2}, \ldots, x_{n}\right)$,

$$
\nabla^{2} \equiv \frac{\partial^{2}}{\partial x_{1}^{2}}+\frac{\partial^{2}}{\partial x_{2}^{2}}+\cdots+\frac{\partial^{2}}{\partial x_{n}^{2}}
$$

\footnotetext{
' Department of Engineering Mechanics, University of Kentucky, Lexington, Kentucky 40506, U.S.A. (C) Copyright Australian Mathematical Society 1984, Serial-fee code 0334-2700/84
} 
and, throughout this paper, summation is implied on repeated indices $\alpha$ and $\sigma$ only. This differential equation, especially in the form (1.2), is the governing equation for a variety of boundary value problems which occur frequently in mathematical physics and engineering analysis. Specifically, if

$$
\kappa \equiv k^{2} \text { with } k=\omega / c, n=1,2 \text { or } 3
$$

where $\omega$ is a (real) frequency and $c$ is a wave speed, then (1.2) is the "reduced" wave equation (or Helmholtz equation) governing time harmonic scalar wave propagation in a non-dissipative medium. Alternatively, with the choice

$$
\kappa \equiv-\lambda^{2}, \quad n=2 \text { or } 3
$$

(1.2) becomes a so-called "modified” Laplace equation which governs a variety of phenomena such as flame spread in a burning solid ([3], [7]) or flow of water in a leaky aquifer ([6], [10]). Also, (1.2) with (1.5) is identical with the Laplace transform of the classical scalar wave equation or the classical heat conduction equation under transient conditions, provided $\lambda$ is identified (e.g. [15]) in terms of the transform parameter and relevant physical parameters. Finally, the choice $\kappa=0$ reduces (1.2) to the ordinary Laplace equation.

Integral equation treatments of specific problems corresponding to particular choice of $\kappa$ (e.g. [10], [15], [2], [12]) have been popular for some time for reasons of computational efficiency and accuracy ( $c f$. remarks in [20], [16]). In most cases, however, formulations are tied closely to the particular problems at issue, and numerical techniques employed to solve the integral equations are varied, often elementary, and even crude by current standards. Further, while three-dimensional solutions exist (e.g. [12], [24]), the majority are limited to plane problems $(n=2)$.

The purpose of the present paper is first to view and formulate a Boundary Integral Equation (BIE) for all problems governed by (1.2) as a class. This BIE as obtained is valid for exterior and interior domains and for $n=3$ as well as $n=2$. Next, we describe a modern systematic process of numerical solution of the general BIE based on the use of "isoparametric" boundary elements. These elements are curvilinear triangles or quadrilaterals for $n=3$ and curvilinear line elements for $n=2$, for which the element geometry and the functions defined over the elements are represented as piecewise-quadratic polynomials. Further, for $n=2$, we introduce certain "singular" shape functions which are useful when there is a singularity in one of the unknown boundary functions at certain points on the boundary of the relevant domain. Finally, we illustrate the entire BIE solution process with three numerical examples drawn from rather diverse physical situations. Specifically, we solve a plane-wave-scattering problem for $n=2$ and a potential-flow problem for $n=3$. Both problems involve potentials, one complex and the other real, defined in regions exterior to a simple, smooth, closed 
surface. Such problems for exterior domains are particularly well suited to a BIE treatment, as opposed to field numerical methods, since only the boundaries need be discretized and conditions at infinity are easily handled analytically. The third example is a heat-conduction problem for $n=2$ interior to a wedge-shaped domain, at one point of which there is an integrable singularity in heat flux. This problem, of some current practical importance [1], is solved for two values of the "wedge angle" using the mentioned singular shape functions. The latter provide for overall solution efficiency and accuracy of representation near the singularity.

\section{BIE formulation}

In the following, regard (1.2) as the governing differential equation for a sufficiently smooth function $\phi$ defined in a region $B$ of two $\left(E^{2}\right)$ - or three $\left(E^{3}\right)$-dimensional space with closed surface $S$. (While a BIE formulation for spaces $E^{n}, n>3$, presents little difficulty, the numerical process to be described would be cumbersome, at best, to implement for such spaces, and we confine attention here to $n=2$ and 3.) Next, let $K(x, y ; \kappa)$ be the fundamental solution (cf. [8]) or free-space Green's function of (1.2), with the property

$$
\nabla^{2} K+\kappa K=\delta(x-y)
$$

where $x \equiv\left(x_{1}, x_{2}\right)$ or $\left(x_{1}, x_{2}, x_{3}\right), y \equiv\left(y_{1}, y_{2}\right)$ or $\left(y_{1}, y_{2}, y_{3}\right)$ are two points in $B$ and $\delta(x-y)$ is the Dirac delta function. Now application of Green's theorem, with attention to the properties of the delta function (and an appropriate limit process as $x \in B \rightarrow x \in S$ ), leads ( $c f$. [16]) to the integral expression

$$
c(x) \phi(x)=\int_{S}\left[\phi(y) K_{\nu}(x, y ; \kappa)-\phi_{\nu}(y) K(x, y ; \kappa)\right] d S(y) .
$$

In (2.2) the subscript $\nu$ indicates the (outward) normal derivative of the function at $y \in S$, and the coefficient $c(x)$ has the values unity for $x \in B$ and zero for $x$ in the region exterior to $B$. If $x \in S, c(x)$ may be obtained from (2.2) by choosing $\phi$ equal to a known elementary solution of (1.2). When a unique tangent (plane) exists at $x \in S$ it is easily shown that $c(x)$ equals one half. If $B$ is the region exterior to a closed surface $S, \phi$ and $\phi_{\nu}$ are assumed to behave, for indefinitely large $y$, such that the integrals in (2.2) taken over an indefinitely large sphere vanish ( $c f$. [13], page 144). In general, $S$ is assumed sufficiently regular ( $c f$. [9]) to permit use of Green's theorem, and for $x \in S$ the integrals, though improper since $K$ is singular for $y=x$, may be shown to exist.

Equation (2.2) may be regarded as an exact integral restatement of a boundary value problem governed by (1.2). Indeed, the BIE strategy for the solution of such problems is as follows. Place $x \in S$, determine $c(x)$, and prescribe (well posed) 
parts of the function pair $\left\{\phi(y), \phi_{\nu}(y)\right\}$ on $S$; solve (2.2) for the unprescribed parts of that pair; place $x \in B$ and evaluate the (now known) integrals (2.2) to obtain the desired solution $\phi(x)$. Gradients of $\phi$ at $x \in B$ may be obtained by differentiation of the integrands in (2.2); note that only the known functions $K$ and $K_{\nu}$ are affected, since they alone are functions of $x$ on the right side of (2.2).

\section{Numerical procedures}

Since a strictly analytical process to carry out the described BIE strategy is out of the question for any practical problem, consider the following numerical solution procedure. Let $S$ of $B, B \in E^{3}$, be discretized into a number $M$ of curvilinear surface elements of either quadrilateral or triangular shape, and locate eight or six nodes, respectively, on each element. Half of the nodes are at the vertices and the other half are on the curvilinear edges of each element, the latter usually located midway between vertex nodes. The coordinates $y_{i}^{\alpha}$ of each node are specified, and the coordinates of a non-nodal point of an element are to be given by

$$
y_{t}(\xi)=M^{\alpha}(\xi) y_{i}^{\alpha}, \quad \alpha=1,2, \ldots, 6 \text { or } 8 ; \quad i=1,2,3,
$$

in which $M^{\alpha}(\xi)$ are second-order shape functions of intrinsic coordinates $(\xi) \equiv$ $\left(\xi_{1}, \xi_{2}\right)$ of the type used in finite element analysis (see e.g. [25] and Appendix). The geometry of $S$ is thus approximated by a system of piecewise-quadratic curvilinear elements coincident at the nodes. For $B \in E^{2}, S$ is a curve or union of several curves, so that in (3.1) $\alpha=1,2,3 ; i=1,2$; and $\xi$ is a scalar variable. Next, assume that $\phi$ or $\phi_{\nu}$ in (2.2) varies over the elements according to

$$
\psi(\xi)=M^{\alpha}(\xi) \psi^{\alpha}
$$

where $\psi^{\alpha}$ is the nodal value of the variable at issue, so that $\phi$ and $\phi_{\nu}$ are thus approximated by the same piecewise quadratic polynomials over the elements as those used to represent the geometry of the elements themselves. This is called an isoparametric representation.

Using this representation, (2.2) for $x \in S$ becomes

$$
\begin{aligned}
c\left(x_{\beta}\right) \phi\left(x_{\beta}\right)= & \phi^{\alpha \sigma} \int_{\gamma} M^{\alpha}(\xi) K_{\nu}\left[x_{\beta}, y(\xi) ; \kappa\right] J^{\sigma}(\xi) d \xi \\
& -\phi_{\nu}^{\alpha \sigma} \int_{\gamma} M^{\alpha}(\xi) K\left[x_{\beta}, y(\xi) ; \kappa\right] J^{\sigma}(\xi) d \xi
\end{aligned}
$$

in which $\sigma=1,2, \ldots, M$ and $\beta=1,2, \ldots, N$, where $N$ is the total number of nodes. The quantities $\psi^{\alpha \sigma}$ mean " the value of $\psi$ at local node $\alpha$ on surface element 
$S_{\mathrm{o}}$ ", and $J^{\mathrm{o}}(\xi)$ is the ratio $d S_{\mathrm{o}}(y) / d \xi$ for the element $S_{\mathrm{o}}$. Note that (3.1) maps $S_{\mathrm{o}}$ to the standard shape $\gamma$ which is either a square or equilateral triangle in the space $\xi_{1} \xi_{2}$ for $B \in E^{3}$. For $B \in E^{2}, \gamma$ is simply the interval -1 to 1 .

The integrals in (3.3) involve only known integrands, and, although certain of them are improper when $x_{\beta} \in S_{0}$, all are integrable either analytically or by Gaussian quadrature following removal of the singularity via transformation (see e.g. [16] or [17] for details). Then, adopting a global designation for $\psi^{\alpha \sigma}$ and letting $x_{\beta}$ occupy all of the nodes, (3.3) may be viewed as a square $N \times N$ system of linear algebraic equations of the form

$$
\mathbf{A} \phi=\mathbf{B} \phi_{\nu},
$$

in which the square matrices $\mathbf{A}$ and $\mathbf{B}$ contain the known integrals (and the $c\left(x_{\beta}\right)$ term) in (3.3). The columns $\phi$ and $\phi_{\nu}$ contain the globally numbered nodal values of the original function pair $\left\{\phi, \phi_{\nu}\right\}$. Thus our solution strategy requires that $\mathbf{A}$ and $B$ be calculated, an appropriate part of the pair $\left\{\phi, \phi_{\nu}\right\}$ be specified, and (3.4) be solved for the nonspecified part of that pair. Once this is done, an interior form of (3.3) is used to generate $\phi(x)$ pointwise at selected $x \in B$ to complete the solution process. Often, however, only the boundary solution is of interest, such that nonspecified $\phi$ or $\phi_{\nu}$ or, perhaps, $d \phi / d S$ are all that are required. This last quantity is easily obtainable using the fact that $\phi(y)$ is represented in terms of $\phi$ and the shape functions.

In some problems, as is the case with the third of our example problems to follow, there is an integrable singularity in the first derivatives of $\phi$ (and thus in $\phi_{\nu}$ ) at one or more isolated points on the boundary $S$. In such a problem, representation of $\phi_{\nu}$ by means of ordinary shape functions ( $c f$. (3.2) and A.1) may be insufficient. Special shape functions are introduced in such a case as follows.

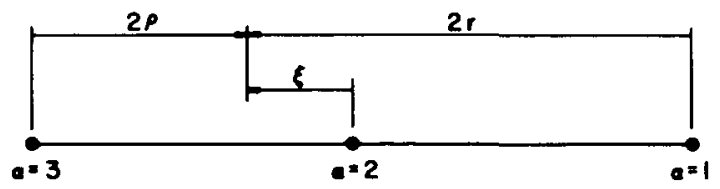

Figure 1. Standard boundary element in $E^{2}$.

Consider a standard element $\gamma$ in $E^{2}$, with local nodes $\alpha=1,2,3$ and intrinsic variable $\xi$ as shown in Figure 1. Suppose the function $\phi_{\nu}(\xi)$ has a singularity at node 1 of known order $s$ such that we may define a special shape function of the form

$$
M_{s}^{1}(r)=A r^{s}+B r^{2}+C r+D, \quad 2 r \equiv 1+\xi
$$


with the requirements that for

$$
\begin{array}{ll}
r \rightarrow 0, & M_{s}^{\prime}(r) \rightarrow \infty, \\
r=\frac{1}{2}, & M_{s}^{1}(r)=0, \\
r=1, & M_{s}^{\prime}(r)=0 .
\end{array}
$$

These requirements may be satisfied by choosing

$$
A=1, \quad B=4\left(\frac{1}{2}\right)^{s}-2, \quad C=1-4\left(\frac{1}{2}\right)^{s}, \quad D=0
$$

so that in terms of $\xi, M_{s}^{1}$ has the form

$$
M_{s}^{\prime}(\xi)=\left(\frac{1}{2}\right)^{s}\left[(1+\xi)^{s}-\left(1-\xi^{2}\right)\right]-\frac{1}{2} \xi(1+\xi) .
$$

Thus if $\phi_{\nu}(\xi)$ has a singularity of order $s$ at node 1, then replacing $M^{1}(\xi)$ in (A.1) by $M_{s}^{1}(\xi)$ as given by (3.8) will result in a more accurate representation of $\phi_{\nu}(\xi)$ over $\gamma$. Note that the quantity $\phi_{\nu}^{\prime}$ in the representation $\phi_{\nu}(\xi)=M^{\alpha}(\xi) \phi_{\nu}^{\alpha}$ is no longer the value of $\phi_{\nu}$ at node 1 under these circumstances. Rather, $\phi_{\nu}^{\prime}$ here is a finite-valued constant expressible in terms of the coefficient of the power of $r$ representing the singularity (e.g. term in parenthesis in (6.7)).

With the introduction of $M_{s}^{\prime}(\xi)$, it is clear from (3.3) that it is now necessary to evaluate integrals of the form

$$
I^{1}=\int_{-1}^{1} M_{s}^{1}(\xi) K\left[x_{\beta}, y(\xi) ; \kappa\right] J^{\sigma}(\xi) d \xi
$$

as ingredients in the matrix $\mathbf{A}$ of (6.12). The "singular" part of this integral is

$$
I_{s}^{\prime}=\int_{-1}^{1}(1+\xi)^{s} K\left[x_{\beta}, y(\xi) ; \kappa\right] J^{\sigma}(\xi) d \xi
$$

in which the singularity in $K$ is logarithmic as $\xi \rightarrow x_{\beta}$. Four cases of interest occur: $x_{\beta}$ occupies none of the nodes on $\gamma$, and $\beta=1,2,3$, i.e. $x_{\beta}$ at each of the three nodes on $\gamma$. For the first case, (3.10) may be integrated by parts, and for the range of $s$ of interest in our third example (cf. (6.7) et seq.) the resulting integrand in the remaining integral is nonsingular and may be evaluated by Gaussian quadrature as usual. For $x_{\beta}$ occupying the nodal positions, an integration by parts together with the transformations

$$
\begin{array}{ll}
\xi=1-\zeta^{2}, & \alpha=1, \\
\xi=\zeta^{2} \text { or } \xi=-\zeta^{2}, & \alpha=2, \\
\xi=\zeta^{2}-1, & \alpha=3,
\end{array}
$$

again produces non-singular integrands. Transformations (3.11) are used according as $x_{\beta}$ occupies the indicated positions (for $\alpha=2, \xi=\zeta^{2}$ is used for integration from nodes 1 to 2 and $\xi=-\zeta^{2}$ for integration from nodes 2 to 3). Again, ordinary Gaussian quadrature may be used. 
Modifications associated with a singular shape function $M_{s}^{3}(\xi)$ to be used if the singularity occurs at node 3 are almost identical to the above. By choosing an element endpoint (node 1 or 3 ) to coincide with a singularity, one need never be concerned with a shape function $M_{s}^{2}(\xi)$.

\section{A plane-wave-scattering problem}

Let a rigid circular cylinder of unit radius be surrounded by a scalar-wavepropagating medium $B$, of infinite extent, with wave-propagation speed $c$. Further, let $\phi$ be the (complex) velocity potential (e.g. [20]) for time-harmonic waves in $B$ of frequency $\omega$, such that

$$
\nabla^{2} \phi+k^{2} \phi=0
$$

must hold throughout $B$, where the wave number $k \equiv \omega / c$. Moreover, we assume that $\phi$ satisfies the Sommerfeld radiation conditions ([13], page 144). Now let $\phi_{0}$ be the potential for a plane harmonic wave incident on the cylinder such that the cylinder axis is parallel to the plane of the incident wave. We seek the total wave potential $\phi$ in $B$, which is the sum $\phi_{0}+\phi_{s}$, where $\phi_{s}$ is the field scattered from the cylinder.

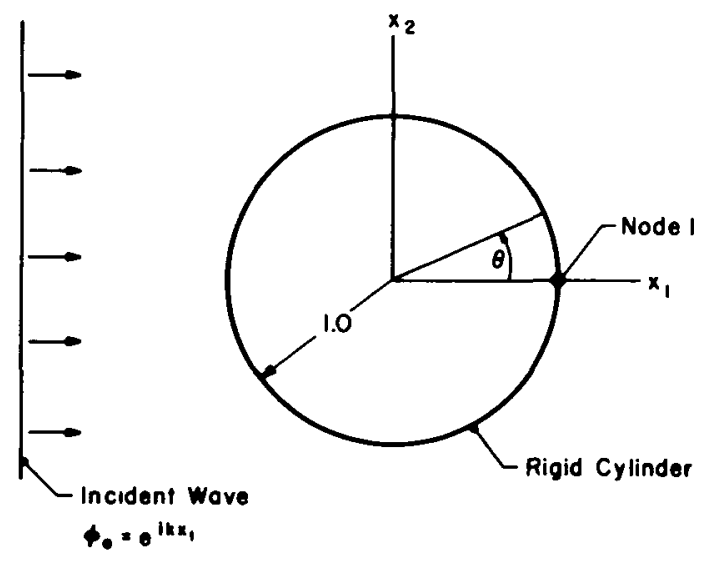

Figure 2. Plane wave scattering problem.

All variables in this problem, as posed, are independent of $x_{3}$, the direction of the cylinder axis, so that the problem becomes one in $E^{2}$. The circular boundary $S$, the coordinate system, and the incident wave are as shown in Figure 2. The necessary function $K(c f .(2.1))$ for (4.1) in $E^{2}$ is

$$
K(x, y ; k)=\frac{i}{4}\left[H_{0}(k r)\right], \quad i=\sqrt{-1}
$$


with $r \equiv|y-x|$, and $H_{0}$ is the Hankel function of the first kind of order zero. Therefore, including the effects of $\phi_{0}$ directly ( $\left.c f .[13]\right),(2.2)$ becomes explicitly

$$
c(x) \phi(x)=\phi_{0}(x)+\frac{i}{4} \int_{S}\left[\phi(y) H_{0}(x, y ; k)-\phi_{\nu}(y) H_{0}(x, y ; k)\right] d S(y)
$$

where $c(x)$ is 1 or $\frac{1}{2}$ according as $x \in B$ or $x \in S$, respectively. For the rigid cylinder, $\phi_{\nu}(y)=0$, so that the second integral in (4.3) vanishes, and we seek $\phi(y)$.

Now, as discussed in Section 3, the approximate form of (4.3) is

$$
A \phi=\phi_{0}
$$

in which $\phi$ and $\phi_{0}$ are the nodal values of the unknown surface potential and the known incident plane-harmonic potential, respectively. The matrix $\mathbf{A}$ is as described in (3.4). Specifically, we first discretize the circle $S$ into $M=9$ equal-length curvilinear elements and $N=18$ nodes, i.e., a node is located on $S$

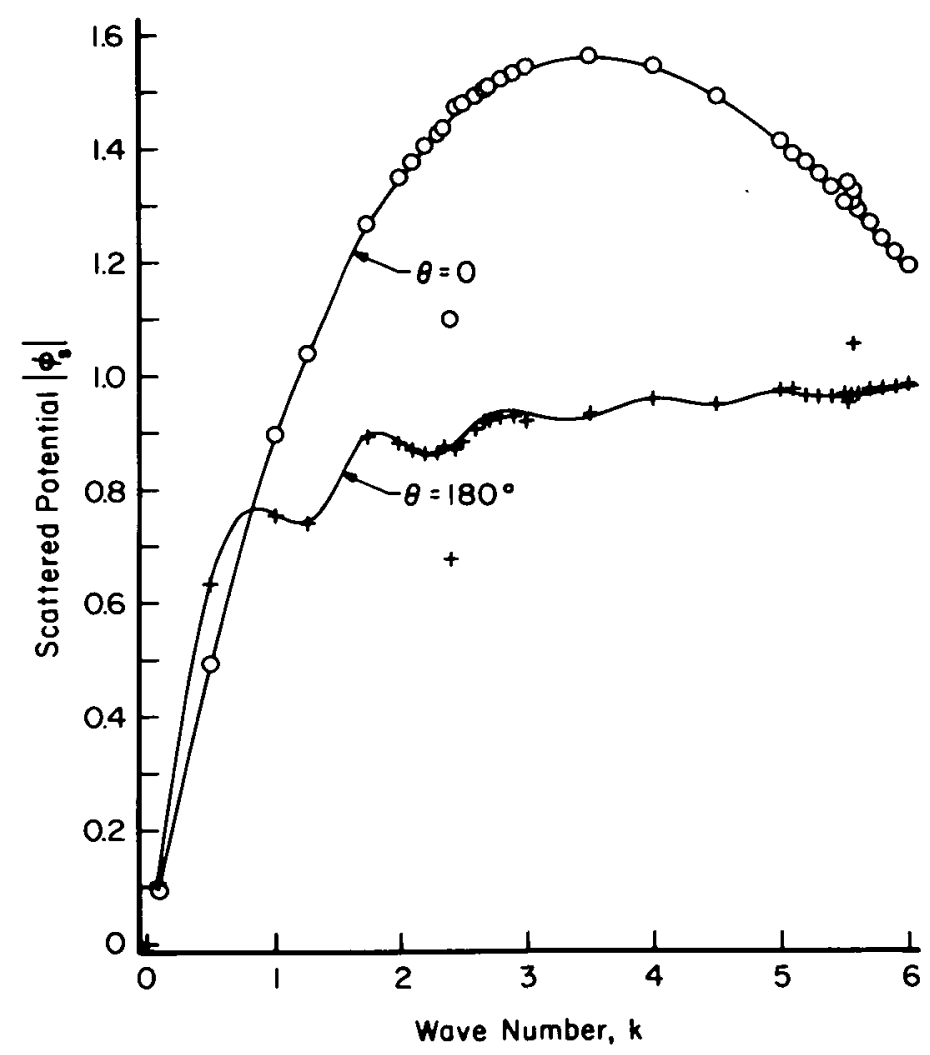

Figure 3. Scattered potential as a function of wave number. 
at equal intervals of $20^{\circ}$ beginning with node 1 on the $x_{1}$ axis. This discretization is used for all chosen values of $k$ up to $k=3.0$. For values of $k>3.0, N=36$ nodes is used, i.e. a node every $10^{\circ}$ on $S$. Higher values of $k$ require increased resolution for accuracy, as might be expected.

Data are obtained for nodes at $\theta=0^{\circ}$ and $\theta=180^{\circ}$, and the magnitude $\left|\phi_{s}\right| \equiv\left(\Re \phi_{s}^{2}+\mathscr{G} \phi_{s}^{2}\right)^{1 / 2}$ of the scattered potential is plotted in Figure 3 as a function of $k$ for these two positions. The solid curve represents the analytical solutions, and the $(O)$ and $(+)$ represent the BIE data. Results as shown are excellent except for data in the vicinity of $k=2.405$ and $k=5.572$, which are wave numbers corresponding to eigenfrequencies of the "associated interior problem" under the soft $(\phi=0)$ boundary condition ( $c f$. [5]). Inaccurate BIE data are expected at or very near these values of $k$, since any integral formula of the type (4.3) is known to break down at such wave numbers. It is possible to reformulate the scattering problem (e.g. [19], [11]) to circumvent the difficulties with the mentioned eigenfrequencies, but such reformulation is beside the purpose of the present paper. However, we find that with the present procedures, ordinary accuracy is obtainable at values of $k$ which are two to three percent away from the eigenvalue, so that serious difficulty with this "fictitious eigenfrequency" phenomenon is not, in fact, encountered. Further, the reciprocal of the condition number of the matrix of coefficients A drops noticeably in size very near the eigenvalues. This could be used as a guide to the acceptability of computed data near an eigenvalue in problems where that eigenvalue may be $a$ priori unknown.

\section{A potential flow problem}

To illustrate the solution process for a problem in $E^{3}$, consider the flow of an ideal fluid past a rigid sphere of radius $a$. For flow in the positive $x_{3}$ direction the well known expression (e.g. [18]) for the velocity potential $\phi$ is

$$
\phi=U\left(1+a^{3} / 2 R^{3}\right) R \cos \theta
$$

in which $R$ is the distance from the sphere's centre to an arbitrary field point $x, \theta$ is the angle between $R$ and the $x_{3}$ axis and $U$ is the undisturbed or free-stream velocity. The potential $\phi$ satisfies Laplace's equation

$$
\nabla^{2} \phi=0
$$

in the region $B$ exterior to the spherical surface $S$, with $\phi_{\nu}=0$ on $S$ itself.

Now we seek a BIE solution for the function $\phi^{*}=\phi-U R \cos \theta$ where on $s$, $\phi_{\nu}^{*}=U(R / a) \cos \theta$. We choose to work with $\phi^{*}$ explicitly rather than $\phi$, since $\phi^{*}$ 
satisfies the required conditions at infinity whereas $\phi$ does not. The necessary function $K(c f .(2.1))$ for (5.2) in $E^{3}$ is

$$
K(x, y)=1 / 4 \pi r(x, y)
$$

such that (2.2) explicitly becomes

$$
c(x) \phi_{r}^{*}(x)=\frac{1}{4 \pi} \int_{S}\left[\phi_{\nu}^{*}(y) \frac{1}{r(x, y)}-\phi^{*}(y)\left(\frac{1}{r(x, y)}\right){ }_{\nu}\right] d S(y)
$$

where again $r=|y-x|, c(x)=1$ or $\frac{1}{2}$ according as $x \in B$ or $x \in S$, respectively, and in which we specify $\phi_{v}^{*}=U(R / a) \cos \theta$.

Under a discretization of $S$ as discussed in Section 3 the approximate form of (5.4) may be written as

$$
\mathbf{A} \boldsymbol{\phi}^{*}=\mathbf{b}^{*}
$$

where $\mathbf{b}^{*} \equiv \mathbf{B} \phi_{v}^{*}$ is a known vector ( $\left.c f(3.4)\right)$, and $\phi^{*}$ and $\phi_{v}^{*}$ represent nodal values of the functions $\phi^{*}$ and $\phi_{v}^{*}$, as before. Here we consider two discretizations of the spherical surface $S$ into curvilinear triangular elements. The first discretization divides $S$ into octants, so that $M=8$ elements with $N=18$ nodes. The second discretization uses a symmetrical subdivision of each octant into three smaller, congruent, curvilinear triangular elements, such that $M=24$ elements with $N=50$ nodes. For each discretization $\phi^{*}$ is obtained as the solution of (5.5).

For the cruder discretization, $\phi^{*}$ departs from the corresponding analytical values by about $13 \%$. At points $x \in B$ at distances from 1.5 a to $100 \mathrm{a},(5.4)$ (with $c(x)=1$ ) yields $\phi^{*}$ values averaging $20 \%$ error. Using the finer discretization these errors are reduced to $3 \%$ and $4 \%$ respectively. Note that $M=24$, though finer than $M=8$, is still a somewhat crude discretization of a spherical surface. We find both sets of accuracy figures surprisingly good (cf. [12], [4] using plane triangle elements) and suggestive of the computational efficiency of the isoparametric representation for curved surfaces in three dimensions.

\section{A moving-flame, heat-conduction problem}

The following is a basic model for a class of problems in flame-spread analysis with application to fire control ([7], [1]). Consider an indefinitely long, infinitely wide slab of combustible solid material of finite thickness which is burning at one end. A typical plane section of the slab is shown in Figure 4a. The flame is confined to the inclined faces of the wedge-shaped end, and the burning process causes the wedge shape to move downward at a uniform speed $V_{F}$ while the wedge angle $\alpha$ remains constant. 


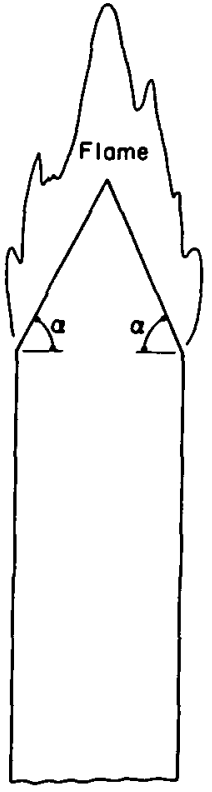

(a)

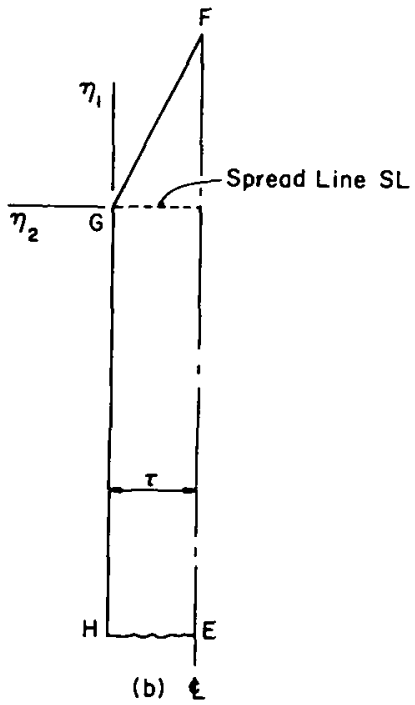

(b) $\varepsilon$

Figure 4. Burning slab model.

Allowing axes of spatial coordinates $\eta_{t}(i=1,2)$ to move with the flame, the governing differential equation in non-dimensional form for the thermal field throughout the slab (e.g. [1]) is

$$
\nabla^{2} \theta=P \frac{\partial \theta}{\partial \eta_{1}}, \quad \nabla^{2} \equiv \frac{\partial^{2}}{\partial \eta_{1}^{2}}+\frac{\partial^{2}}{\partial \eta_{2}^{2}}
$$

where $\theta$ is the non-dimensional temperature, and $P$ is the so-called Péclet number. This number is known in terms of the slab half-thickness $\tau, V_{F}$, and other thermal parameters. We need consider only a half-thickness of the slab as shown in Figure $4 \mathrm{~b}$, and from symmetry there is zero heat flow across the centre-line $E F$.

The specific boundary value problem here is to find $\theta$ throughout the finite plane region $E F G H$ under the boundary conditions

$$
\begin{array}{ll}
\theta_{\nu}=0 & \text { on edges } E F, G H, \\
\theta=1 & \text { on edge } F G, \\
\theta=0 & \text { on edge } H E .
\end{array}
$$


Of particular interest in this problem is the heat flux $Q$ across the spread line $(S L)$, i.e.,

$$
Q=\int_{S L} \frac{\partial \theta}{\partial \eta_{1}} d \eta_{1} \equiv P \int_{S L} \theta d \eta_{1}
$$

where the second integral expression for $Q$ follows from Green's theorem.

Now $(6.1)$ can be easily put in the form $(1,2)$ through the transformation

$$
\theta=\exp \left(p \eta_{1}\right) \psi, \quad P \equiv 2 p
$$

such that in terms of the transformation function $\psi,(6.1)$ becomes

$$
\nabla^{2} \psi-p^{2} \psi=0
$$

with

$$
\begin{array}{ll}
\psi_{\nu}=0 & \text { on edges } E F, G H, \\
\psi=\exp \left(-p \eta_{1}\right) & \text { on edges } F G, \\
\psi=0 & \text { on edge } H E,
\end{array}
$$

as the corresponding boundary conditions.

The boundary value problem in the form (6.5), (6.6) is now ready for a BIE treatment as in the preceding sections, but it should be noted that a singularity in the function $\psi_{v}$ exists at the point $G$ for $\alpha>0$ (Figure 4). An elementary eigenvalue analysis ( $c f$. [22], [23]) reveals that for sufficiently small $r$ near $G$, we may obtain the order of singularity in $\psi_{\nu}$ from

$$
\psi(r, \hat{\theta})=r^{\lambda}\left(c_{1} \sin \lambda \hat{\theta}+c_{2} \cos \lambda \hat{\theta}\right),
$$

where $(r, \hat{\theta})$ are polar coordinates of a point in $B$ or $S$ near $G, c_{1}$ and $c_{2}$ are constants, and $\lambda$ has the value $\pi / 2 \beta$ with $\beta=\pi / 2+\alpha$ (Figure 4). Clearly then, $\psi$ has an unbounded gradient for $\pi / 2<\beta<2 \pi$ at $r=0$. Physically, this implies that the local heat flux $q$, which is proportional to $\psi_{\nu}$ at any boundary point, is undefined at $G$. This singularity, however, is integrable, so that while attention is required to represent this singularity with special shape functions (cf. (3.5) et seq.) with attendant modifications in the numerical process, the analytical BIE statement of the problem may proceed as outlined in Section 2.

The necessary function $K(c f .(2.1))$ for (6.5) in $E^{2}$ is

$$
K(x, y ; k)=\frac{1}{2 \pi} K_{0}(x, y ; p)
$$

where $K_{0}$ is the modified Bessel function of the second kind of order zero. The BIE formula, i.e. (2.2), now has the explicit form

$$
2 \pi c(x) \psi(x)=\int_{S}\left[\psi_{\nu}(y) K_{0}(x, y ; p)-\psi(y) K_{0}(x, y ; p)\right] d S(y)
$$


where $S$ is the boundary of the region in Figure $4 \mathrm{~b}$, i.e. the union of the line segments $E F G H$. Here $c(x)=1$ for $x \in B$, where $B$ is the region interior to $S$, and $c(x)=\frac{1}{2}$ for $x \in S$, except when $x$ occupies the "corner" points, $E, F, G$, and $H$. At these points $c(x)$ equals the interior angle (in radians). However, $c(x)$ for any $x \in S$ may be obtained without explicit attention to the corner points, since the function

$$
\psi^{*} \equiv \exp \left(p \eta_{1}\right)
$$

is an elementary solution of (6.5) and thus satisfies (6.9) as well. Substituting $\psi^{*}$ into (6.9) and solving for $c(x)$ we obtain

$$
c(x)=\frac{1}{2 \pi} \int_{S}\left\{\frac{\exp \left(p \eta_{1}(y)\right)}{\exp \left(p \eta_{1}(x)\right)}\left[p K_{0}(x, y ; p)-K_{0}(x, y ; p)\right]\right\} d S(y)
$$

which is used for all computations of $c(x), x \in S$, including $x$ at the corner points. This approach for $c(x)$ provides more systematic and accurate computation of improper integrals which arise following discretization of (6.9) than would separate calculation of interior angles at corners ( $c f .[15])$.

In the present problem, boundary data (see (6.6)) are mixed such that the unknown functions in (6.9) are $\psi(y)$ on edges $E F$ and $G H$ and $\psi_{\nu}(y)$ on edges $F G$ and $H E$. Under a discretization of $S,(6.9)$ has the form

$$
\mathbf{A u}=\mathbf{v}
$$

in which $\mathbf{u}$ is the vector of unknown values of $\psi$ and $\psi_{\nu}$ at nodes on $S$, except, of course, for the value of $\psi_{v}$ at point $G$, which is undefined. (See comment following (3.8).) Also $\mathbf{v}$ is the vector of known nodal values obtained from integrals of prescribed (non-zero) data, and $\mathbf{A}$ is the matrix of coefficients of the unknowns, as before. However, here $\mathbf{A}$ includes integrals of special shape functions used to represent $\psi_{\nu}$ over one element on $S$, adjacent to point $G$, at one end of which $\psi_{\nu}$ is singular. Using $\tau=1$ and locating surface $H E 5$ units away from the spread line (Figure $4 \mathrm{~b})$ proved sufficient to employ the $\theta=0(\psi=0)$ boundary conditions on $H E$ for all calculations.

For $\alpha=0$, there is no singularity at point $G$, and the analytical solution in terms of $\theta$ with boundary conditions (6.2) is simply $\theta=\exp \left(p \eta_{1}\right)$. Using $M=16$ elements ( $N=32$ nodes), BIE data for $\theta$ on the boundary and at several interior points compare with analytical values with an error of less than $0.1 \%$. Normalized flux values, $\theta_{\nu}$ on $S$, similarly compare with an error of $0.15 \%$.

For $0<\alpha<\pi / 2$ the situation is complicated by the fact that $\theta_{\nu}$ is singular at point $G$ with order of singularity $r^{\lambda-1}$ (see (6.7) et seq.), and an analytical solution is not available for data verification. The authors [1] were interested in the solution of this problem prior to the development of the singular shape functions 
(SSF) as described in Section 3. It was decided to obtain BIE data, using ordinary shape functions (OSF) (A.1), for $\alpha$ in increments of $10^{\circ}$ from $\alpha=0^{\circ}$ to $\alpha=90^{\circ}$, for a variety of Péclet numbers $P$. Sufficiently fine discretizations (large $M$ with small elements "bunched" near $G$ ) were used to try to obtain meaningful values of $Q(6.3)$ and $\theta$ (along edge $G H$ ), which were of primary interest in their heat transfer studies [1]. It was anticipated that the admitted inadequate representation of the singularity in $\theta_{i v}$ at $G$ using OSF would be minimized with the fine "mesh" near $G$ and that the variable of interest away from $G$ would be little affected. By and large, this proved to be true; $Q$ as a function of $\alpha$ obtained from the BIE analysis agreed well with asymptotic expressions valid for small $\alpha$ and/or large $P$. Further, surface $\theta$ so obtained agreed well with experimental values for $\alpha=80^{\circ}$ and several values of $P$ as shown in detail in [1]. However, despite the success in [1] for the intended purpose there, we were dissatisfied with the "brute force" discretization procedures used and raised the following questions. To what extent could coarser discretizations be used with SSF and maintain desired accuracy of all data? Also, how well could we represent $\theta_{\nu}$ along edge $G F$ using SSF and how bad was the representation using OSF? (The criteria for mesh grading suggested in [14] would undoubtedly improve the performance of the OSF in representing the singularity.)

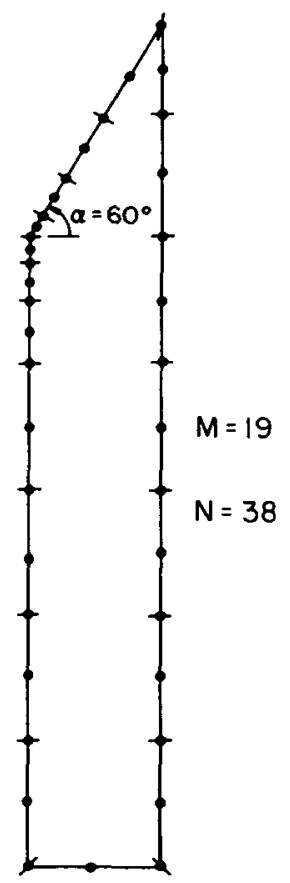

(a)

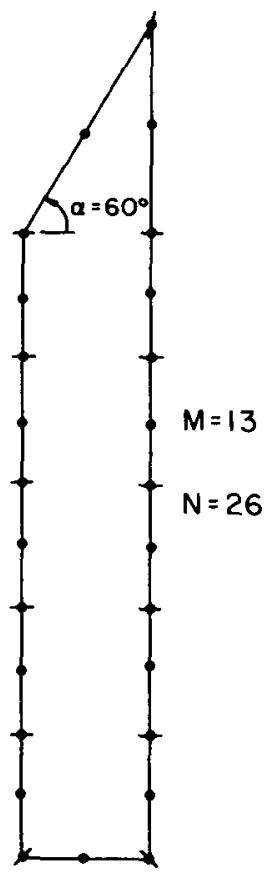

(b)

Figure 5. Two discretizations $\alpha=60^{\circ}$. 


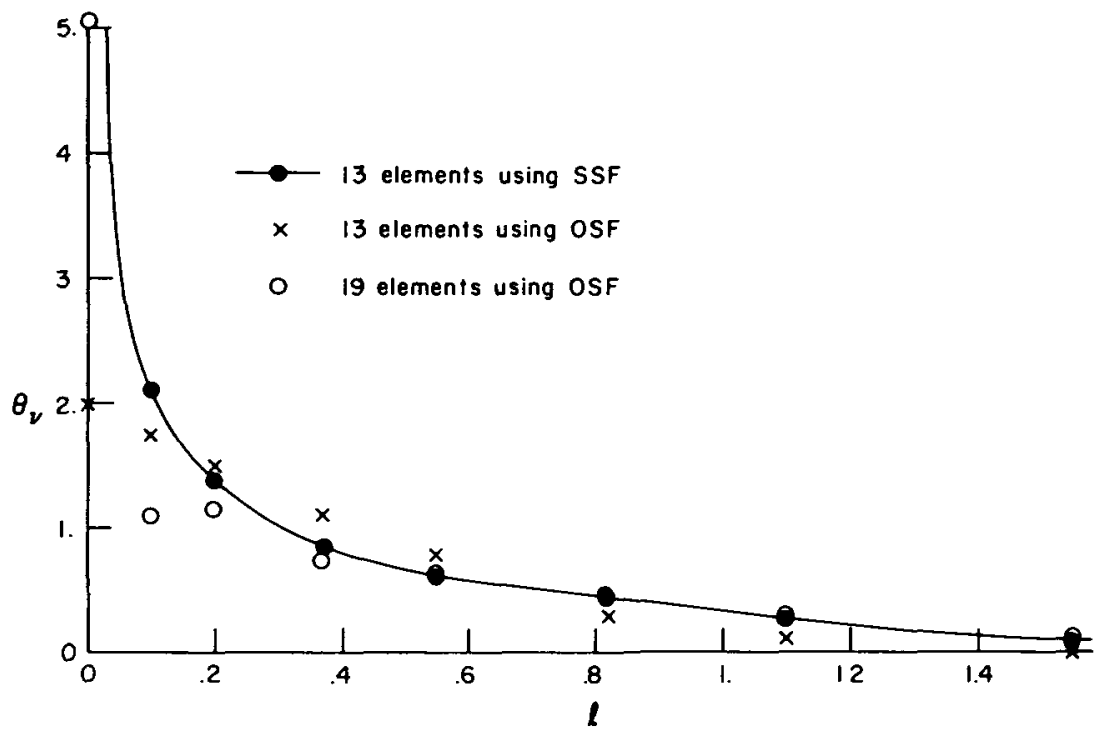

Figure 6. Behavior of $\theta_{v}$ on edge $G F$ near $G$.

To answer these questions, SSF were introduced as indicated in Section 3, and additional BIE solutions were obtained for the $\alpha=60^{\circ}$ and $\alpha=80^{\circ}$ cases, with $P=1$, using successively coarser meshes and comparing accuracy and CPU times using OSF and SSF. It was found that a reduction of $20 \%$ to $30 \%$ in the total number of elements with no need for bunching of elements near $G$ could be achieved using SSF, maintaining comparable accuracy of data overall. For example, Figure 5 depicts two discretizations for $\alpha=60^{\circ}$, the coarser of which yielded virtually identical $Q$ and surface $\theta$ using SSF as did the finer one using OSF. CPU time, mostly dependent on $M$, was reduced accordingly. Moreover, when used with OSF the coarser mesh yielded data differing from the accepted values by as much as $7 \%$ away from the singularity (see Table 1).

Near the singularity, the variation of $\theta_{\nu}$ with distance $l$ from $G$ along edge $G F$ is depicted in Figure 6. The solid curve is the representation using SSF for the known order of singularity for $\alpha=60^{\circ}$. Clearly the representation using OSF is different very near $G$ where the SSF representation approaches the exact representation. Thus through increased accuracy of local representation the SSF seem to provide a measure of overall computation efficiency as well.

Numerical data for the problems in this and the preceding sections were obtained using an IBM 370/165 computer with no advantage taken of any symmetry in $B$ in programming. Execution times for individual problem solutions ranged from 2 to 15 seconds. 
TABLE 1 . The boundary temperature at $\eta_{1}<0$ and $\eta_{2}=0$ for $\alpha=60^{\circ}$ and Péclet number of one.

\begin{tabular}{|c|c|c|c|}
\hline \multirow[b]{2}{*}{$\begin{array}{c}\eta_{1} \\
\left(\eta_{2}=0\right)\end{array}$} & \multicolumn{3}{|c|}{ Boundary temperature $\theta$} \\
\hline & $\begin{array}{c}\text { OSF } \\
\text { Mesh Figure 5a }\end{array}$ & $\begin{array}{c}\text { SSF } \\
\text { Mesh Figure 5b }\end{array}$ & $\begin{array}{c}\text { OSF } \\
\text { Mesh Figure 5b }\end{array}$ \\
\hline-0.5 & 0.4538 & 0.4579 & 0.4845 \\
\hline-1.0 & 0.2636 & 0.2663 & 0.2823 \\
\hline-1.5 & 0.1565 & 0.1581 & 0.1678 \\
\hline$-\hat{L} . \hat{v}$ & $0.0 \hat{9} 2 \hat{\delta}$ & $0.0 \hat{9} 37$ & 0.09996 \\
\hline-2.5 & 0.0544 & 0.0549 & 0.0584 \\
\hline-3.0 & 0.0311 & $0.03+4$ & 0.0334 \\
\hline-3.5 & 0.0169 & 0.0171 & 0.0182 \\
\hline-4.0 & 0.0084 & 0.0084 & 0.0090 \\
\hline-4.5 & 0.0032 & 0.0032 & 0.0034 \\
\hline
\end{tabular}

\section{Acknowledgements}

Portions of this work were supported by a grant from the National Science Foundation, grant number: NSF CME-8013461. Thanks are due also to S. Lahiri for gathering certain numerical data, to $\mathrm{G}$. Fairweather for a valuable conversation, and to separate referees for calling references [14] and [11] to the authors' attention.

\section{Appendix}

Ordinary Shape Functions

(a) One-Dimensional $\left(E^{2}\right)$

$$
M^{1}(\xi)=-\frac{1}{2}\left(\xi-\xi^{2}\right), \quad M^{2}(\xi)=1-\xi^{2}, \quad M^{3}(\xi)=\frac{1}{2}\left(\xi+\xi^{2}\right) .
$$

(b) Quadrilateral $\left(E^{3}\right)$

$$
\begin{array}{lll}
M_{R}^{1}(\xi)=\frac{1}{4}\left(\xi_{1}+1\right)\left(\xi_{2}+1\right)\left(\xi_{1}+\xi_{2}-1\right), & M_{R}^{5}(\xi)=\frac{1}{2}\left(\xi_{1}+1\right)\left(1-\xi_{2}^{2}\right), \\
M_{R}^{2}(\xi)=\frac{1}{4}\left(\xi_{1}-1\right)\left(\xi_{2}+1\right)\left(\xi_{1}-\xi_{2}+1\right), & M_{R}^{6}(\xi)=\frac{1}{2}\left(\xi_{2}+1\right)\left(1-\xi_{1}^{2}\right), \\
M_{R}^{3}(\xi)=\frac{1}{4}\left(1-\xi_{1}\right)\left(\xi_{2}-1\right)\left(\xi_{1}+\xi_{2}+1\right), & M_{R}^{7}(\xi)=\frac{1}{2}\left(\xi_{1}-1\right)\left(\xi_{2}^{2}-1\right), \\
M_{R}^{4}(\xi)=\frac{1}{4}\left(\xi_{1}+1\right)\left(\xi_{2}-1\right)\left(\xi_{2}-\xi_{1}+1\right), & M_{R}^{8}(\xi)=\frac{1}{2}\left(1-\xi_{2}\right)\left(1-\xi_{1}^{2}\right) .
\end{array}
$$

(c) Triangular

$$
\begin{array}{ll}
M_{T}^{1}(\xi)=\xi_{1}\left(2 \xi_{1}-1\right), & M_{T}^{4}(\xi)=4 \xi_{1} \xi_{3}, \\
M_{T}^{2}(\xi)=\xi_{2}\left(2 \xi_{2}-1\right), & M_{T}^{5}(\xi)=4 \xi_{1} \xi_{2}, \quad \xi_{1}+\xi_{2}+\xi_{3}=1, \\
M_{T}^{3}(\xi)=\xi_{3}\left(2 \xi_{3}-1\right), & M_{T}^{6}(\xi)=4 \xi_{2} \xi_{3} .
\end{array}
$$




\section{References}

[1] R. A. Altenkirch, M. Rezayat, R. Eichhorn and F. J. Rizzo, "A study of heat conduction forward of flame spread in solids; by the boundary integral equation method", Trans. ASME Ser. C J. Heat Transfer 104 (1982), 734-740.

[2] R. P. Banaugh and W. Goldsmith, "Diffraction of steady acoustic waves by surfaces of arbitrary shape", J. Acoust. Soc. Amer. 35 (1963), 1590-1601.

[3] H. S. Carslaw and J. C. Jaeger, Conduction of heat in solids (Oxford University Press, London, 1967).

[4] L. H. Chen and D. G. Schweikert, "Sound radiation from an arbitrary body", J. Acoust. Soc. Amer. 35 (1963), 1626-1632.

[5] L. G. Copley, "Fundamental results concerning integral representations in acoustic radiation", J. Acoust. Soc. Amer. 44 (1968), 28-32.

[6] Roger J. M. DeWiest, "Flow to an eccentric well in a leaky circular aquifer with varied lateral replenishment", Geofis. Pura Appl. 54 (1963), 90-102.

[7] A. E. Frey and J. S. T'ien, "A theory of flame spread over a solid fuel including finite-rate chemical kinetics", Combustion and Flame 36 (1979), 263-289.

[8] F. John, Plane waves and spherical means (Interscience, New York, Ist edition, 1955).

[9] O. D. Kellogg, Foundatins of potential theory (Dover, New York, 1st edition, 1953).

[10] O. E. Lafe, "Boundary integral solutions to nearly horizontal flows in multiply zoned aquifers", Ph.D. Thesis, Cornell University, 1981.

[11] P. A. Martin, "On the null-field equations for the exterior problems of acoustics", Quart. J. Mech. Appl. Math. 33 (1980), 385-396.

[12] W. L. Meyer, W. A. Bell, B. T. Zinn and M. P. Stallybrass, "Boundary integral solutions of three dimensional acoustic radiation problems", J. Sound and Vibration 59 (1978), 245-262.

[13] Y. H. Pao and Ch. Ch. Mow, Diffraction of elastic waves and dynamic stress concentrations (Crane, Russak and Company Inc., New York, 1973).

[14] J. R. Rice, "On the degree of convergence of nonlinear spline approximation", in Approximation with special emphasis on spline functions (ed. I. J. Schoenberg), (Academic Press, New York, 1969).

[15] F. J. Rizzo and D. J. Shippy, "A method of solution for certain problems of transient heat conduction", AIAA J. 8 (1970), 2004-2009.

[16] F. J. Rizzo and D. J. Shippy, "The boundary integral equation method with application to certain stress concentration problems in elasticity", J. Austral. Math. Soc. Ser. B 22 (1981), $381-393$.

[17] F. J. Rizzo and D. J. Shippy, "An advanced boundary integral equation method for three-dimensional thermoelasticity", Internat. J. Numer. Methods Engrg. 11 (1977), 1753-1768.

[18] H. Rouse (ed.), Advanced mechanics of fluids (John Wiley and Sons, Inc., New York, 1969).

[19] H. A. Schenck, "Improved integral formulation for acoustic radiation problems", J. Acoust. Soc. Amer. 44 (1968), $41-58$.

[20] R. P. Shaw, "Boundary integral equation methods applied to wave problems", in Developments in boundary element methods-1, Chapter 6, 121-154.

[21] S. L. Sobolov, Partial differential equations of mathematical physics (Pergamon Press, London, 1964).

[22] M. Stern, "On calculating thermally induced stress singularities", Conference on thermal stresses in severe environment (Plenum Press, 1980), 123-134.

[23] M. L. Williams, "Stress singularities resulting from various boundary conditions in angular corners of plates in extension", J. Appl. Mech. 74 (1952), 526-528.

[24] Y. S. Wu, "The boundary integral equation method using various approximation techniques for problems governed by Laplace's equation", Master's thesis, University of Kentucky, 1976.

[25] O. C. Zienkiewicz, The finite element method (McGraw-Hill Book Company (UK) Limited, London, 3rd edition, 1977). 